For reprint orders, please contact: reprints@futuremedicine.com

\title{
Decades research and implementation science of HIV prevention, treatment and cure: highlights from Symposium 2017
}

\begin{abstract}
Pirapon June Ohata ${ }^{*, 1}$, Kesdao Nanthapisal', Chavalun Ruengpanyathip', Pornwinit Sattayamong', Anchalee Avihingsanon ${ }^{1,2}$, Sivaporn Gatechompol', Torsak Bunupuradah', Akarin Hiransuthikul', Stephen J Kerr', Tanya Do', Thanyawee Puthanakit ${ }^{1,3}$, Eugene Kroon ${ }^{4}$, Donn Colby ${ }^{4}$, Reshmie Ramautarsing ${ }^{5}$, Steve Kraus ${ }^{6}$, Brianna Harrison ${ }^{6}$, Taoufik Bakkali ${ }^{6}$, Opass Putcharoen ${ }^{7}$, Kiat Ruxrungtham ${ }^{1,2}$ \& Praphan Phanuphak ${ }^{1,4,5}$, on behalf of the Symposium Team
\end{abstract}

The 19th Bangkok International Symposium on HIV Medicine, Bangkok, Thailand, 18-20 January 2017

This training is one of the most established and largest, with up-to-date information provided by well-renowned international speakers and hence considered one of the best in the Asia Pacific region. The Symposium is offered every third week of January for three full days. Celebrating HIV Netherlands Australia Thailand research collaboration's 20th anniversary, for the first time, the sessions were covered real-time through webcasts, streamed live via the internet. Speakers included community advocates voicing and addressing certain issues, and the entire third day was dedicated to symposiums. HIV Netherlands Australia Thailand research collaboration continues to strive to provide well-rounded trainings of quality to the region's professional healthcare workers, hoping to significantly impact the delivery of health services. Noteworthy sessions have been briefly summarized in this report.

First draft submitted: 30 January 2017; Accepted for publication: 2 February 2017;

Published online: 28 April 2017

Special Lecture: Third Professor Joep Lange \& Ms. Jacqueline van Tongeren Memorial Lecture: same-day ART: caution to the wind?

Diane Havlir (HIV/AIDS Division, San Francisco General Hospital and University of California, San Francisco, CA, USA) stated that physicians should consider offering same-day antiretroviral therapy (ART) to all patients to reduce reservoir size, preserve the immune system and prevent transmission. However, the patients' readiness (i.e., nonadherence resulting in drug resistance), provider readiness (i.e., have all necessary information to be able to select the best/optimal regimen for the patient) and system readiness (i.e., capacity of the clinic) to start ART should also be assessed to avoid causing harm to the patient. Four large randomized trials

'HIV-NAT, Thai Red Cross AIDS Research Centre (TRCARC), 104 Ratchadamri Rd, Pathumwan, Bangkok 10330, Thailand 2Division of Allergy \& Immunology, Faculty of Medicine, Chulalongkorn University, Rama IV Road, Pathumwan, Bangkok 10330, Thailand ${ }^{3}$ Department of Pediatrics, Faculty of Medicine, Chulalongkorn University, Rama IV Road, Pathumwan, Bangkok 10330, Thailand ${ }^{4}$ SEARCH, Thai Red Cross AIDS Research Centre (TRCARC), 104 Ratchadamri Rd, Pathumwan, Bangkok 10330, Thailand 5Prevention Unit, Thai Red Cross AIDS Research Centre (TRCARC), 104 Ratchadamri Rd, Pathumwan, Bangkok 10330, Thailand

${ }^{6}$ Regional Support Team, Asia \& the Pacific, UNAIDS Asia Pacific RST, 12th Floor, UN Building, Rajdamnern-nok Avenue, Phra Nakorn, Bangkok 10200, Thailand

Division of Infectious Diseases, Department of Medicine, Faculty of Medicine, Chulalongkorn University, Rama IV Road, Pathumwan, Bangkok 10330, Thailand

*Author for correspondence: Tel.: +662 652 3040; Fax: +662 252 5779; juneohata4@gmail.com

\section{KEYWORDS}

- acute infection - HIV

- implementation research

Future
Medicine 
conducted in South Africa, Uganda, Haiti and USA, showed that same-day ART is not only doable but also feasible, yielding better clinical health outcomes and linkages to care for those who start early [1-3].

\section{Addressing stigma \& discrimination in healthcare settings}

Nadia Rasheed (HIV, Health \& Development Asia \& the Pacific, UNDP, Thailand), Taweesap Siraprapasiri (National AIDS Management Center, Department of Disease Control [DDC], Ministry of Public Health [MOPH], Thailand), Sumet Ongwandee (Bureau of AIDS, TB and STIs, DDC, MOPH, Thailand), Suwat Chariyalertsak (Research Institute for Health Sciences [RIHES], Chiang Mai University, Thailand), Bounpheng Philavong (Centre for HIV/AIDS and STI, Ministry of Health of Lao PDR, Lao PDR) and Niwat Suwannapattana (National Community Advisory Board, Thailand) informed the participants about existing stigma and discrimination (S\&D) situations in various healthcare settings within the region which deleteriously undermine the effectiveness of HIV investments and responses as well as HIV healthcare and its related services. Thailand has developed a tool to measure the S\&D levels in a hospital setting with six domains, consisting of fear of HIV infection, over self-protection, observed discrimination, attitudes toward HIV-infected staff, attitudes toward people living with HIV (PLHIV) and attitudes toward key populations (men who have sex with men, transgenders, female sex workers, people who inject drugs and migrant workers), to measure the $S \& D$ levels in a hospital setting. This tool has been translated and culturally adjusted and used in Vietnam and Lao PDR. The results have been surprising because it showed that many professional healthcare workers had higher S\&D levels when compared with nonprofessional healthcare worker and lacked education in HIV, exacerbating the $S \& D$ against people infected with HIV. Issues and strategies (i.e., Generic National Code of Conduct for Hospitals) to rectify this were thoroughly discussed with the aim to reduce S\&D of patients with HIV and key populations by $90 \%$ by 2030 [4] .

\section{Thorough information on acute HIV infection (day 2)}

Jintanat Ananworanich (US Military HIV Research Program, USA and SEARCH, Thai Red Cross AIDS Research Centre [TRCARC],
Thailand) defined acute HIV infection and introduced the techniques available in resourcelimited settings used to diagnose it [5]. She emphasized the urgency to identify and treat acute HIV because it will reduce the size of the HIV reservoirs [6], preserve the immune system and prevent transmission [7]. Infants have a higher chance of eradicating HIV because their immune system is less active, respond better to vaccines, have more naive CD 4 cells that are resistant to HIV and fewer central memory CD4 cells [8]. Nicolas Chomont (Centre de Recherche du Chum [CRCHUM], Canada) followed that with the definition of latent reservoirs. There are three main strategies for 'cure': to limit the establishment of the reservoir by starting treatment early and make the uninfected cells resistant to HIV [9]; to reduce the size of the reservoir by depleting the infected cells and use the 'shock and kill' technique [10] and to control the reservoir by using vaccines and immunotherapy [10]. Serena Spudich (Yale University School of Medicine, USA) corroborated that the CNS serves as one of HIV's reservoirs [11] and provided examples of how scientists are trying to eradicate the reservoir from the brain, reiterating the need to start treatment early and concomitant use of HDAC-inhibitors and other strategies such as the use of vorinostat, hydroxychloroquine and maraviroc. Lydie Trautmann (US Military HIV Research Program, USA) focused on immune therapies that may eradicate HIV in people with acute infections (i.e., decrease inflammation [12], inject potent antibodies [13] and boost $\mathrm{T}$ - and $\mathrm{B}$-cell responses with vaccine [14]). On the other hand, Sharon Lewin (Doherty Institute for Infection and Immunity, Australia) concentrated on immune therapies for people with chronic infection, who started ART late. These strategies are similar to those with acute infection such as the use of latency reversing agents such as TLR7 agonists, reduce inflammation/proliferation (for example with IL21 superagonist, or antiproliferation agents Sirolimus and Everolimus, or with agents that disrupt other inflammatory pathways). Irini Sereti (NIH, NIAID, USA) emphasized the impact of late presentation and late ART such as developments of opportunistic infections (i.e., cytomegalovirus, TB, cryptococcal meningitis, HCV, HPV) [15], HIV-related neoplasia (i.e., lymphoma, Kaposi sarcoma), non-AIDS complications (i.e., cardiovascular 
disease-atherosclerosis, neuroinflammation), severity of the inflammation and larger establishments of the reservoir. Aside from the clinical perspective, Gail Henderson (Department of Social Medicine, UNC School of Medicine, USA) elucidated reasons why people agreed to participate in HIV cure trials despite the anticipated risks. From her cohort, through the nested Decision-Making Study, being off ART was seen as a clear benefit, psychologically and socially even though they knew that they likely would have to eventually restart ART. They anticipated that the information/science/knowledge gathered from the study would benefit others/society, and wanted to 'give back' to society. Therefore, in order to have useful clinical data, it is also important that researchers understand and address study participants' needs because they are a crucial part of any clinical trial.

\section{Recent HIV clinical researches \& their potential clinical applications}

David A Cooper (The Kirby Institute for Infection and Immunity in Society, Australia) stated that the SMART study provided information on the detrimental effects of treatment interruption [16], while the START study provided information that treatment should be started early in adults [17], which was supported also by the HPTN052 findings [18]. The ENCORE-1 study informed that lower dose of Efaverenz (EFV; $400 \mathrm{mg}$ ) was as effective and efficacious as the standard dose of $600 \mathrm{mg}$ when used in Asians [19], and another study known as the LASA showed that $200 \mathrm{mg}$ Atazanavir (ATV) among treatment-stable patients was not inferior to the standard dose $300 \mathrm{mg}$ ATV but had fewer side effects in Asians with smaller body size and weight [20]. Many of the results obtained from clinical trials have influenced the WHO's and Thai's treatment guidelines.

\section{Symposium 4 on TB: new drugs \\ \& treatment strategies}

For the past few years, the TB field has been stagnant in terms of new drug developments and treatment. However, Nicholas Paton (National University of Singapore [NUS]) described there are now several new drugs in the pipeline [21]. He provided information on bedaquiline (Phase III), delamanid (Phase III), linezolid (Phase II) and meropenem ( $\beta$-lactams repurposed for multidrug resistant TB). The most exciting part of the talk concerned new approaches to combat MDR$\mathrm{TB}$ [22] to reduce the treatment duration from 40 weeks to 16 weeks by using the drugs previously mentioned. In addition, he raised the possibility that the treatment duration for drug sensitive TB may go from 6 months to 2 months or 10 days by increasing the dosage (i.e., high-dose rifampicin), increasing adherence via directly observed therapy, short course (i.e., Mobile Interactive Supervised Therapy, also known as the MIST system; smartphone-based system to measure and support adherence to TB treatment), using adjunct therapy to increase the immune response (i.e., blocking IL-4 or using pascolizumab) and revising the treatment modality (i.e., TRUNCATE-TB Trial will assess the 2 month regimens using novel combinations to augment treatment effectiveness for drug-sensitive TB; this trial will be conducted at HIV Netherlands Australia Thailand research collaboration).

\section{Symposium 5 on viral hepatitis: ending $\mathrm{HCV}$}

$\mathrm{HCV}$ is curable so the question was raised why there were 1.45 million deaths/year worldwide in 2013, up by 63\% from 1990? Juergen Rockstroh (University of Bonn, Germany) briefly went over HCV epidemiology and its clinical outcome. The most distressful information was the fact that globally, only $5 \%$ of the 170 million HCVinfected people are aware of their infection. Hence, WHO has provided guideline with target to eliminate HCV by 2030 [23]. Data from Egypt and Georgia showed that elimination is feasible and possible. Barriers to achieving elimination in other countries are attributed to the cost of treatment and role of generics, restricted treatment access or treatment only for $\geq \mathrm{F} 3$, many people undiagnosed or not linked to care and lack of preventative measures (i.e., opiate substitution treatment, needle and syringe programs). Despite being cured, many tend to be reinfected with HCV. Among IDUs/prisoners and HIV/HCV co-infected patients, the reinfection rates were 13.2 and $21.7 \%$, respectively. Thus vaccines for $\mathrm{HCV}$ are greatly needed. Elimination of HCV is possible but requires all of the stakeholders to work together to increase testing, provide high quality harm reduction, improve quality of health services, increase access to treatment, have a preventative vaccine and eliminate $S \& D$.

The 20th Bangkok International Symposium on HIV Medicine will be held on 17-19 January 2018. 


\section{Acknowledgements}

The authors would like to thank all of the speakers and moderators for their continued support in providing the latest information to the Symposium. The authors would like to thank GSK ViiV for supporting the webcasts/live streaming of the Symposium. We are extremely grateful to all of our sponsors, collaborators and partners to make this event possible. The authors would also like to thank the participants who attended the event and provided feedback so we can work on improving the program. This Symposium would not have been possible without the dedicated hard work and strong commitment from the Symposium team for developing the scientific program to organizing, coordinating and running the show.

Financial \& competing interests disclosure The 19th Bangkok International Symposium on HIV Medicine was supported by the Thai Red Cross AIDS Research Centre (TRCARC), Chulalongkorn University,
Thailand Convention and Exhibition Bureau (TCEB), GILEAD, LF Asia, GlaxoSmithKline (GSK), ViiV Healthcare, Mylan Atlanta Medicare, Vela Diagnostics, MSD, Alere, abbvie, Government Pharmaceutical Organization (GPO), Roche and amfAR/TREAT Asia. $K$ Ruxrungtham has received the Senior Research Scholar from Thailand Research Fund (TRF). K Ruxrungtham received honoraria or consultation fees from Merck, Roche, Jensen-Cilag, Tibotec, Mylan and GPO (Thailand). He also has participated in a company sponsored speaker's bureau from Abbott, Gilead, Bristol-Myers Squibb, Merck, Roche, Jensen-Cilag, GlaxoSmithKline and GPO. The authors have no other relevant affliations or financial involvement with any organization or entity with a financial interest in or financial conflict with the subject matter or materials discussed in the manuscript apart from those disclosed.

No writing assistance was utilized in the production of this manuscript.

\section{References}

1 Rosen S, Fox MP, Larson BA et al. Accelerating the uptake and timing of antiretroviral therapy initiation in sub-Saharan Africa: an operations research agenda. PLoS Med. 13(8), e1002106 (2016).

2 Amanyire G, Semitala FC, Namusobya J et al. Effects of a multicomponent intervention to streamline initiation of antiretroviral therapy in Africa: a steppedwedge cluster-randomised trial. Lancet HIV3(11), e539-e548 (2016).

3 Koenig S, Dorvil N, Severe P et al. Same-day HIV testing and antiretroviral therapy initiation results in higher rates of treatment initiation and retention in care. Presented at: 21st International AIDS Conference. Durban, South Africa, 18-22 July 2016.

4 Agenda for Zero Discrimination in Health Care.

www.unaids.org/

5 Mayaphi SH, Martin DJ, Quinn TC et al. Detection of acute and early HIV-1 infections in an HIV hyper-endemic area with limited resources. PLoS One 11(10), e0164943 (2016).

6 Ananworanich J, Chomont N, Eller LA et al. HIV DNA set point is rapidly established in acute HIV infection and dramatically reduced by early ART. EBioMedicine 11, 68-72 (2016).

7 Robb ML, Eller LA, Kibuuka $\mathrm{H}$ et al. Prospective study of acute HIV-1 infection in adults in east Africa and Thailand. $N$. Engl. J. Med. 374(22), 2120-2130 (2016).

8 Ananworanich J, Puthanakit T, Suntarattiwong $\mathrm{P}$ et al. Reduced markers of HIV persistence and restricted HIV-specific immune responses after early antiretroviral therapy in children. AIDS 28(7), 1015-1020 (2014).

9 Tebas P, Stein D, Tang WW et al. Gene editing of CCR5 in autologous CD $4 \mathrm{~T}$ cells of persons infected with HIV. N. Engl. J. Med. 370 (10), 901-910 (2014).

10 Fromentin R, Bakeman W, Lawani MB et al. $\mathrm{CD}^{+} \mathrm{T}$ cells expressing PD-1, TIGIT and LAG-3 contribute to HIV persistence during ART. PLoS Pathog. 12(7), e1005761 (2016).

11 Sturdevant CB, Joseph SB, Schnell G, Price RW, Swanstrom R, Spudich S.

Compartmentalized replication of $\mathrm{R} 5$ T cell-tropic HIV-1 in the central nervous system early in the course of infection. PLoS Pathog. 11(3), e1004720 (2015).

12 Kim CJ, Walmsley SL, Raboud JM et al. Can probiotics reduce inflammation and enhance gut immune health in people living with HIV: study designs for the Probiotic Visbiome for Inflammation and Translocation (PROOV IT) pilot trials. HIV Clin. Trials 17(4), 147-157 (2016).

13 Scheid JF, Horwitz JA, Bar-On Y et al. HIV-1 antibody 3BNC117 suppresses viral rebound in humans during treatment interruption. Nature 535(7613), 556-560 (2016).
14 Borducchi EN, Cabral C, Stephenson KE et al. Ad26/MVA therapeutic vaccination with TLR7 stimulation in SIV-infected rhesus monkeys. Nature 540 (7632), 284-287 (2016).

15 Sereti I, Folkers GK, Meintjes G, Boulware DR. Towards a scalable HIV cure research agenda: the role of co-infections. J. Virus Erad. 1(4), 269-271 (2015).

16 Friis-Moller N, Reiss P, Sabin CA et al. Class of antiretroviral drugs and the risk of myocardial infarction. N. Engl. J. Med. 356(17), 1723-1735 (2007).

17 O'Connor J, Vjecha MJ, Phillips AN et al. Effect of immediate initiation of antiretroviral therapy on risk of severe bacterial infections in HIV-positive people with CD 4 cell counts of more than 500 cells per muL: secondary outcome results from a randomised controlled trial. Lancet HIV doi:10.1016/S2352-3018(16)30216-8 (2017) (Epub ahead of print).

18 Eshleman SH, Hudelson SE, Redd AD et al. Treatment as prevention: characterization of partner infections in the HIV prevention trials network 052 trial. J. Acquir. Immune Defic. Syndr. 74(1), 112-116 (2017).

19 Dickinson L, Amin J, Else L et al. Pharmacokinetic and Pharmacodynamic comparison of once-daily efavirenz ( $400 \mathrm{mg}$ vs. $600 \mathrm{mg}$ ) in treatment-naive HIVinfected patients: results of the ENCORE1 study. Clin. Pharmacol. Ther. 98(4), 406-416 (2015). 
20 Bunupuradah T, Kiertiburanakul S, Avihingsanon A et al. Low-dose versus standarddose ritonavir-boosted atazanavir in virologically suppressed Thai adults with HIV (LASA), a randomised, open-label, non-inferiority trial. Lancet HIV3(8), e343-e350 (2016).
21 Working Group on New TB drugs. Stop TB partnerships. Accelerating discovery. www.newtbdrugs.org

22 WHO Treatment Guidelines for Drug Resistant Tuberculosis 2016 Update. WHO,
Geneva, Switzerland (2016). http://apps.who.int/

23 World Health Organization. Combating hepatitis $\mathrm{B}$ and $\mathrm{C}$ to reach elimination by 2030 (2016).

http://apps.who.int/ 